\title{
Viabilidade de duas novas técnicas para inseminação intrauterina laparoscópica em ovinos
}

[Two new techniques for laparoscopic intrauterine insemination in sheep - feasibility study]

\author{
J.P.S. Feranti ${ }^{1 *}$, M.V. Brun ${ }^{1}$, E. Zanella ${ }^{2}$, S.A. Messina ${ }^{3}$, R. Schuh ${ }^{2}$, \\ F.R. Santos ${ }^{4}$, G. Brambatti ${ }^{4}$ \\ ${ }^{1}$ Universidade Federal de Santa Maria (UFSM) - Santa Maria, RS \\ ${ }^{2}$ Universidade de Passo Fundo (UPF) - Faculdade de Agronomia e Medicina Veterinária (FAMV) -Passo Fundo, RS \\ ${ }^{3}$ Universidade de Passo Fundo (UPF) - (FAMV) - Passo Fundo, RS *In memoriam \\ ${ }^{4}$ Universidade Federal do Rio Grande do Sul - Favet - Porto Alegre, RS
}

\section{RESUMO}

Avaliaram-se duas novas técnicas de inseminação laparoscópica intrauterina com o uso de agulha espinhal em ovinos. Foram realizadas quatro etapas experimentais, sendo os animais separados em dois grupos em cada etapa. Para tanto, foram utilizados os posicionamentos quadrupedal e dorsal. Realizou-se a inseminação de 80 ovinos após a sincronização de cio. Verificou-se que os acessos laparoscópicos propostos foram viáveis para a inseminação em ovinos. Considerou-se que a inseminação em posicionamento quadrupedal foi tecnicamente mais difícil se comparada ao acesso laparoscópico em decúbito dorsal, porém, com o aprimoramento da técnica e na dependência dos resultados quanto aos índices de prenhez, poderá se tornar procedimento adequado para a inseminação de ovelhas.

Palavras-chave: ovelhas, videolaparoscopia, agulha espinhal, útero, prenhez

\begin{abstract}
This study evaluated two new laparoscopic intrauterine insemination techniques with spinal needles in sheep. Four experimental stages were done, in which the animals were separated into two groups aiming to evaluate the techniques used. We used a total of 80 estrus-synchronized sheep, which were placed in quadrupedal or dorsal recumbence. The results showed that both insemination techniques using a spinal needle are feasible, but the quadrupedal insemination is technically more difficult compared to the laparoscopic approach in the dorsal position. With technical improvement and depending on the results in relation to pregnancy rates, this access could be considered an alternative procedure for sheep insemination.
\end{abstract}

Keywords: sheep, laparoscopy, spinal needle, uterus, pregnancy

\section{INTRODUÇÃO}

Evidencia-se cada vez mais o crescimento na criação e na produção de ovelhas, bem como a ampliação no mercado de carne ovina, influenciados pelo investimento em pesquisas relacionadas ao melhoramento genético. Uma das maneiras de se intensificar o melhoramento é o uso de biotécnicas da reprodução, entre elas a inseminação artificial, a qual proporciona maior amplitude de resultados, já que poucos reprodutores selecionados produzem sêmen suficiente para a grande quantidade de fêmeas que reproduzem anualmente (Oliveira, 2009).
O uso da inseminação artificial pode acelerar o melhoramento genético, facilitar a realização de testes de progênie, evitar a transmissão de doenças venéreas, viabilizar a obtenção de produtos de reprodutores alojados em outras regiões ou países (até mesmo de animais que já morreram) e possibilitar que machos com subfertilidade adquirida produzam descendentes (Oliveira, 2009). Além disso, permite que o produtor multiplique os genótipos dentro do rebanho, com o uso de sêmen de diferentes animais comprovadamente melhoristas, sem ampliar o número de reprodutores no plantel. Ainda, torna possível o maior controle

Recebido em 23 de novembro de 2011

Aceito em 12 de dezembro de 2012

E-mail: johny.sf@hotmail.com 
zootécnico do rebanho, entre outras condições, pela identificação de fêmeas que não apresentaram estro e pelo maior controle de partos com datas precisas (Oliveira, 2009). A inseminação artificial intensifica o manejo reprodutivo (Bicudo et al., 2000), principalmente quando aliada ao uso da criopreservação do sêmen.

Os resultados obtidos com a deposição de sêmen congelado na vagina são pobres quando comparados com a colocação deste diretamente na luz uterina de ovinos (Hontou, 1993). Rotineiramente, realiza-se a aplicação dos espermatozoides no interior do útero próximo ao período de ovulação, a fim de ampliar as taxas de gestação, haja vista o curto período de sobrevivência dessas células (Salamon e Maxwell, 1995).

A cérvix da ovelha é longa, fibrosa e tubular. Caracteriza-se por possuir espessa parede e luz constrita, com o canal cervical formado por várias proeminências de forma transversa ou espiralada, com saliências fixas conhecidas como anéis anulares (de três a oito anéis), medindo cerca de 4 a $7 \mathrm{~cm}$ de comprimento (Silva et al., 2009). Essa anatomia dificulta a passagem da pipeta de inseminação pela cérvix, sendo um dos principais fatores que limita a propagação da inseminação artificial na espécie ovina (Oliveira, 2009).

Apesar de a inseminação intrauterina laparoscópica possuir vantagens em relação às outras técnicas, ela traz algumas desvantagens ou dificuldades, como a necessidade de um profissional altamente qualificado, o tempo de inseminação mais prolongado e os custos operacionais elevados devido aos instrumentais necessários para a laparoscopia. Além disso, há riscos de complicações anestésicas decorrentes do pneumoperitônio e da posição de Trendelemburg (Vannozi et al., 2002). Algumas dessas podem estar relacionadas à insuflação da cavidade com dióxido de carbono $\left(\mathrm{CO}_{2}\right)$, o que pode provocar elevação na pressão intraperitoneal e potencial absorção de $\mathrm{CO}_{2}$, podendo levar à elevação significativa da pressão parcial de $\mathrm{CO}_{2}$ e a uma diminuição da pressão parcial arterial de oxigênio (Vannozi et al., 2002). Além disso, existe a ampliação da possibilidade de aspiração do conteúdo de regurgitação ruminal pela posição de
Trendelemburg, condição que pode até mesmo ser fatal (Allen e Borkowski, 1999).

Apesar das vantagens da inseminação laparoscópica em ovinos quando comparada à via transvaginal, com ou sem dilatação cervical e com o uso de fármacos (Stellflug et al., 2001), ainda se justifica a busca por procedimentos minimamente invasivos, que possam ocasionar menor desconforto trans e pós-operatórios, e com adequados resultados em relação aos índices de prenhez. Ressalta-se que, com os melhores protocolos de indução ao cio disponíveis para inseminação intrauterina laparoscópica nessa espécie, pode-se observar gestação em menos de 75\% dos animais (Hill et al., 1998).

O presente trabalho tem o objetivo de verificar a viabilidade de duas novas técnicas de inseminação laparoscópica intrauterina com o uso de agulha espinhal em ovinos, sendo os animais posicionados em decúbito dorsal e em posição quadrupedal.

\section{MATERIAL E MÉTODOS}

Foram realizadas quatro etapas de inseminações (E1), (E2), (E3) e (E4) em períodos distintos, sendo os animais, em cada uma destas, separados em dois grupos (G1 e G2) para a avaliação das técnicas utilizadas. Para tanto, foram operadas 80 ovelhas, da raça Suffolk, com peso estimado de $80 \mathrm{~kg}$, submetidas a dois diferentes protocolos de sincronização de cio: 1) em 42 animais (G1), o protocolo constou da aplicação por pessário de acetato de medroxiprogesterona por 14 dias, sendo, por ocasião da remoção do implante, administrados 300UI de eCG; b) em 38 ovinos (G2), o protocolo constou da aplicação por pessário de acetato de medroxiprogesterona por 12 dias e da administração de $0,25 \mathrm{~mL}$ de terramicina por pessário para prevenção de possíveis infecções vaginais e/ou uterinas. Por ocasião da remoção do implante, administraramse nesses animais 400UI de eCG.

Após o período mínimo de 48 horas e máximo de 52 horas, foram realizadas as inseminações intrauterinas laparoscópicas. No G1, 44 ovinos foram colocados em mesa metálica a qual permitia a manutenção do animal em decúbito dorsal associada ou não ao cefalodeclive (Trendelemburg). No G2, 36 animais foram colocados em um brete de contenção metálica, 
confeccionado especialmente para esse experimento, o qual permitia a contenção do animal em posicionamento quadrupedal (Fig. 1).

Os pacientes receberam como pré-medicação citrato de fentanila, $2 \mu \mathrm{g} . \mathrm{kg}^{-1}$, associado, na mesma seringa, com acepromazina, $0,05 \mathrm{mg} \cdot \mathrm{kg}^{-1}$, aproximadamente 15 minutos antes da operação. Promoveu-se a infiltração analgésica dos tecidos subcutâneo e muscular com lidocaína a $2 \%$, diluída em $\mathrm{NaCl} 0,9 \%, 2 \mathrm{mg} \cdot \mathrm{kg}^{-1}$, nas regiões onde seriam introduzidos os dois portais.

No G1, após a antissepsia do campo operatório e a colocação de campos plásticos, introduziu-se agulha de Veress na linha média ventral por uma incisão promovida na metade da distância entre o púbis e a cicatriz umbilical, a partir da qual a cavidade foi insuflada com $\mathrm{CO}_{2}, 2 \mathrm{~L}$. $\min ^{-1}$, até se obter a pressão de $12 \mathrm{mmHg}$. Após a introdução do primeiro portal $(5 \mathrm{~mm})$ no mesmo local, posicionou-se o paciente em Trendelemburg, realizando-se a introdução do segundo portal sob visualização direta. Um dos cornos uterinos foi apreendido com pinça de Kelly e tracionado ventralmente, permitindo a escolha do local de aplicação do sêmen. Para tanto, empregou-se agulha espinhal descartável (27G x 3 1/2"') posicionada através da pele e da parede muscular até alcançar a luz uterina. Aplicou-se, com seringa hipodérmica de $1 \mathrm{~mL}$, aproximadamente 0,25 mL de sêmen por corno uterino (Fig. 2). Em todos os casos foi utilizado sêmen de único doador, congelado na forma de pellet, com a concentração espermática viável estimada em $50 \times 10^{6} / 0,5 \mathrm{~mL}$.

No G2, após a assepsia cirúrgica e a colocação de campos plásticos, introduziu-se a agulha de Veress na parede abdominal lateral direita por uma incisão promovida na metade da distância entre a linha dorsal e a linha mamária, e entre o ponto médio desde a última costela e a protuberância ilíaca. A partir desse acesso, a cavidade foi insuflada com $\mathrm{CO}_{2}, 2 \mathrm{~L} \cdot \mathrm{min}^{-1}$, até se obter a pressão de $12 \mathrm{mmHg}$. Sequencialmente à introdução do primeiro portal $(5 \mathrm{~mm})$ no mesmo local, realizou-se o posicionamento do segundo portal sob visualização direta. Um dos cornos uterinos foi apreendido com pinça de Kelly e tracionado lateralmente, permitindo a escolha do local de introdução do sêmen. Empregou-se agulha espinhal descartável aplicada através da pele e da parede muscular até alcançar a luz uterina (Fig. 3). Aplicou-se, com seringa hipodérmica de $1 \mathrm{~mL}$, aproximadamente $0,5 \mathrm{~mL}$ de sêmen no corno uterino direito sob visualização direta.

O pós-operatório nas quatro etapas constou da higiene das feridas operatórias com $\mathrm{NaCl} 0,9 \%$, aplicação de piretroide em forma de spray e administração de flunexim meglumine, $1 \mathrm{mg} \cdot \mathrm{kg}^{-1}$, IM, por três dias. Na E1 realizou-se avaliação ultrassonográfica aos 55 dias de pós-operatório; na E2 realizaram-se aos 57 dias, na E3 e na E4 não foram realizadas as avaliações ultrassonográficas, sendo consideradas apenas as análises dos resultados trans e pós-operatórias imediatos.

\section{RESULTADOS E DISCUSSÃO}

Na primeira etapa (E1), na qual todos os animais foram posicionados em decúbito dorsal para a realização das inseminações, os procedimentos foram realizados em $13,76 \pm 7,88 \mathrm{~min} \quad \mathrm{e}$ observaram-se dificuldades técnicas em três ovinos quanto à obtenção do pneumoperitônio com a agulha na primeira tentativa, sendo necessária mais de uma punção com a Veress para se obter a insuflação adequada. Essa condição foi relacionada diretamente com a curva de aprendizado da equipe.

O volume de sêmen de uma palheta por animal foi suficiente para a aplicação intra-uterina, mesmo em um ovino no qual a agulha transpassou o corno uterino e pequena quantidade seminal foi aplicada na cavidade. Nesse animal, a manipulação uterina com a pinça permitiu pronta observação do incorreto posicionamento da agulha. Dos 21 ovinos inseminados, 19 foram utilizados para considerar a taxa de prenhez e dois foram descartados, pois possuíam alterações no tamanho uterino observadas durante a laparoscopia. Um ovino veio a óbito por causa desconhecida vários dias após a inseminação, não podendo se correlacionar o óbito ao procedimento pela ausência de necropsia. Cinco (26,3\%) demonstraram gestação na avaliação ultrassonográfica, sendo que $40 \%$ destes possuíam gestação gemelar, com dois embriões. Considerando-se as gestações gemelares, obtevese a relação entre o número de animais inseminados e o número de fetos produzidos de $36,8 \%$. 


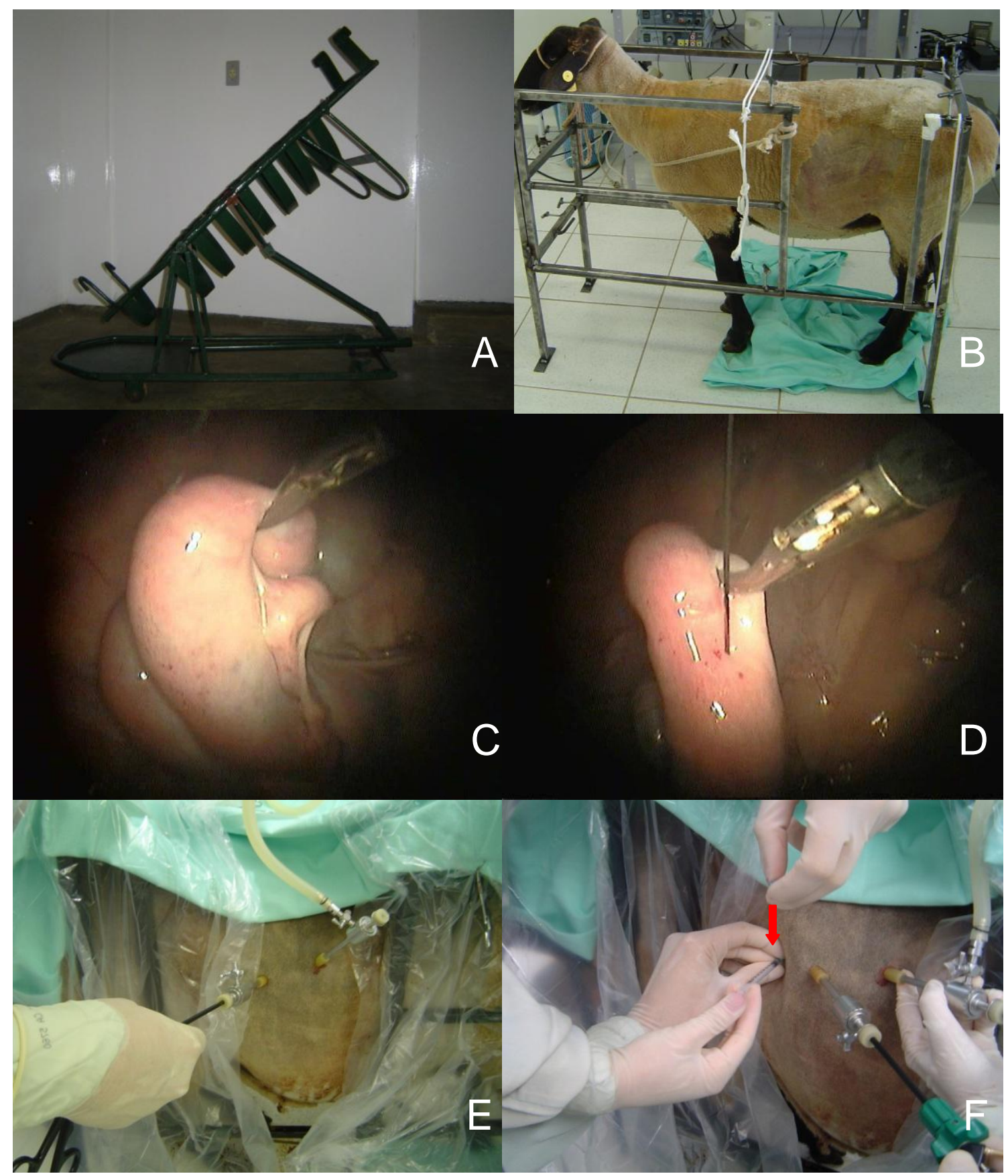

Figura 1. Diferentes aspectos quanto à realização da inseminação em ovinos: (A) mesa metálica para colocação dos animais em decúbito dorsal e cefalodeclive (posição de Trendelemburg) ao se realizar inseminação laparoscópica intrauterina; (B) brete metálico portátil confeccionado para este experimento, o qual permite a contenção do animal em posicionamento quadrupedal; (C) apreensão do corno uterino com pinça de Kelly e seu tracionamento em direção à parede muscular sob visão laparoscópica para a realização das inseminações intrauterinas com agulha espinhal; (D) disposição dos portais de acesso durante a inseminação intrauterina com o animal em posição quadrupedal; (E) aplicação de sêmen com seringa hipodérmica (seta vermelha) acoplada à agulha espinhal descartável através da pele e da parede muscular (F). 
O uso de dois portais com os pacientes em decúbito dorsal é semelhante ao indicado pela maioria dos autores, contudo o posicionamento destes no presente trabalho difere significativamente das descrições rotineiras, tal como descreve Hontou (1993). Em vez de se utilizar um portal por parede abdominal (direita e esquerda), optou-se pela colocação na linha média ventral para minimizar a lesão muscular de acesso. Entretanto, ao se optar por esse acesso, é necessário extremo cuidado para que não ocorram lesões nos vasos mamários que se encontram nessa região.

Na segunda etapa (E2), foram comparados dois acessos diferentes, sendo que no G1 foi realizado de forma similar ao descrito na E1, e no G2 foi obtido o posicionamento quadrupedal e o acesso foi realizado pelo flanco, utilizando-se também dois portais e agulha espinhal. Assim como na primeira etapa do experimento, o volume de sêmen de um pellet por animal foi suficiente para a administração intrauterina, mesmo em um ovino no qual foi aplicada apenas meia dose de sêmen. Dos 21 ovinos que tiveram o cio sincronizado, 18 foram utilizados para considerar a taxa de prenhez. Um ovino da E2(G2) foi descartado, pois possuía alterações no tamanho uterino constatadas durante a laparoscopia. Um animal veio a óbito durante a operação por regurgitação na E2(G1), e outro ovino regurgitou na E2(G1), sendo necessário interrompê-la antes da inseminação.

$\mathrm{Na}$ E2(G2), dos oito animais utilizados, quatro não estavam gestantes (50\%), três apresentavam gestação de um feto $(37,5 \%)$ e um apresentava gestação gemelar $(12,5 \%)$. Nessa etapa, uma ovelha desenvolveu gestação mesmo com aplicação de apenas meia dose do sêmen. Também se observou gestação em outro animal que recebeu subdosagem de hormônio antes da inseminação. $\quad \mathrm{Na}$ E2(G1), em que foram utilizados 10 animais, sete não apresentaram gestação (70\%), três apresentaram gestação com um feto (30\%) e nenhuma gestação gemelar foi diagnosticada. Os diagnósticos gestacionais foram baseados apenas nos exames ultrassonográficos.

Na terceira etapa (E3), em que se utilizou técnica semelhante à da E2(G2), ou seja, com os animais em posição quadrupedal, as inseminações foram realizadas em 17,63 \pm 7,03 min, ocorrendo complicações em três animais. Em uma ovelha não foi possível a realização do procedimento laparoscópico devido ao descolamento do peritônio do músculo transverso após a insuflação, impossibilitando, assim, a visualização do útero e dos ovários e de seus ligamentos e vasos. As outras complicações foram constatadas em dois de quatro animais nos quais se testava um novo acesso de introdução da agulha de Veress para obtenção do pneumoperitônio. Nesse acesso alternativo, obtido com o posicionamento da agulha pelo $12^{\circ}$ espaço intercostal, em uma das ovelhas ocorreu a transfixação do fígado pelo instrumento, condição que não ocasionou nenhuma hemorragia mensurável; e noutra ovelha houve a perfuração do diafragma com consequente pneumotórax, o qual foi manejado por drenagem torácica com escalpe com boa evolução do paciente. Tal posicionamento da Veress é utilizado em equinos (Silva et al., 2001), mas não é usual em ovinos, sendo que, no presente experimento, mostrou-se potencialmente arriscado.

O que difere a E2(G2) da E3 é que o estudo foi desenvolvido em conjunto com o Laboratório de Reprodução do Hospital Veterinário da Universidade de Passo Fundo, no qual se testaram, além da inseminação realizada pelo flanco, dois diferentes diluentes para congelamento de sêmen ovino. Na E3 também se buscou aplicar um protocolo de tranquilização e analgesia que proporcionasse um menor desconforto trans e pós-operatório ao animal, utilizando para isso o bloqueio paravertebral, associado ao local.

$\mathrm{Na}$ quarta etapa (E4), na qual se utilizaram ambos os posicionamentos para a realização das inseminações, em 10 animais do G1 e oito do G2 os procedimentos foram realizados em $22,72 \pm 12,54 \mathrm{~min}$, observando-se complicação apenas em um animal do G2, no qual houve o descolamento do peritônio após a insuflação, sendo necessária a tração do útero até o espaço pré-peritoneal para, então, somente após, proceder à deposição do sêmen da luz do órgão. $\mathrm{O}$ volume de sêmen de uma paleta por animal também foi suficiente para a aplicação intrauterina.

$\mathrm{O} \mathrm{CO}_{2}$ tem sido o gás mais empregado para a obtenção de pneumoperitônio, por ser 
transparente, não comburente, de fácil acesso, baixo custo e altamente solúvel em água (Ortega, 1994). É um produto endógeno natural eliminado pelos pulmões durante a respiração (Morgan, 1994). Porém, é de conhecimento que o $\mathrm{CO}_{2}$ reduz o $\mathrm{pH}$ da superfície peritoneal, provocando mais dor quando comparado com outros gases, fato que implica a utilização de anestesia geral (Lemos et al., 2003). Em inseminações intrauterinas laparoscópicas em campo, o uso da anestesia geral não é prática rotineira. Associado a isso, emprega-se rotineiramente ar ambiental administrado com bomba manual. $\mathrm{O}$ presente trabalho demonstra que o uso de $\mathrm{CO}_{2}$ medicinal a campo, com aplicação das anestesias local e regional, é prática viável e segura para ovinos, contudo torna-se indicado o controle da pressão intracavitária do gás e da velocidade de insuflação, ambas condições que não podem ser ajustadas com a utilização de ar ambiental, além do que a administração dessa combinação de gases pode acarretar em consequências hemodinâmicas sérias para o paciente, sem falar na contaminação cavitária.

Todos os protocolos de tranquilização e analgesia se mostraram adequados para a realização das laparoscopias e estiveram associados a pouco desconforto dos pacientes. Além da necessidade de manutenção do bem-estar dos animais, pensou-se também nos possíveis melhores resultados finais no que se refere aos índices de prenhez com o uso de analgesia preemptiva descrita, uma vez que é comprovado que o estímulo doloroso e o estresse causam aumento de cortisol plasmático, que pode interferir diretamente nos índices reprodutivos (Hill et al., 1998).

Quanto aos índices de manutenção de prenhez, consideram-se os obtidos em E1 e E2(G2) ainda muito baixos, mas ressalta-se que foram utilizados animais que haviam sido descartados da reprodução por não gestarem após monta natural, os quais foram submetidos à inseminação com sêmen congelado. Por essas condições, já se esperavam pobres resultados quanto a esse aspecto. É importante ressaltar que devem ser realizados estudos posteriores utilizando ovinos em boas condições reprodutivas para se verificar a adequação do uso de agulha espinhal nos animais em decúbito dorsal, bem como para se certificar de que o acesso quadrupedal pode (ou não) obter resultados similares aos alcançados pelo decúbito dorsal em relação aos índices de prenhez.

$\mathrm{Na}$ terceira etapa consideraram-se os índices de manutenção de prenhez muito abaixo dos esperados, podendo associar essa condição a diferentes fatores. Entre esses, atribui-se ao estresse a que se submeteram esses pacientes no pré-operatório como uma das principais causas, já que as inseminações foram feitas em ambiente hospitalar no mesmo dia da chegada dos animais da fazenda, os quais foram transportados em veículo inapropriado. Além disso, também foram utilizados animais que haviam sido descartados da reprodução por não gestarem após a monta natural, e o protocolo de sincronização de cio diferenciou-se demasiadamente daqueles das etapas anteriores. Por todos esses motivos, as taxas de prenhez não foram consideradas na comparação entre os grupos.

No presente estudo, priorizou-se testar principalmente os aspectos transoperatórios em relação aos dois acessos propostos, já que o uso da agulha se apresentou como uma nova possibilidade aos princípios da inseminação laparoscópica com os ovinos em decúbito dorsal. De forma similar, a posição quadrupedal, a qual foi desenvolvida pelos próprios autores (Brun et al., 2006), foi proposta como uma nova alternativa, que talvez possa ser até mesmo menos estressante para os pacientes e que poderá estar associada a menores alterações hemodinâmicas que o posicionamento em Trendelemburg.

\section{CONCLUSÕES}

As técnicas laparoscópicas de inseminação intrauterina com agulha espinhal em ovinos são viáveis. Considera-se que a inseminação com o animal em posicionamento quadrupedal com a agulha espinhal é tecnicamente mais difícil se comparada à de acesso laparoscópico em decúbito dorsal. Porém, com o aprimoramento da técnica e, na dependência de resultados quanto aos índices de prenhez, poderá tornar-se uma alternativa para a inseminação de ovelhas. 
Esse trabalho foi realizado após a aprovação do Comitê de Ética em Pesquisa (CEP_UPF) da Universidade de Passo Fundo, sob protocolo ${ }^{\circ}$ 717-2005, e foi financiado parcialmente por bolsa de iniciação científica do $\mathrm{CNPq}$, sob protocolo n $106366 / 2006-8$.

\section{REFERÊNCIAS}

ALLEN, M.J.; BORKOWSKI, G.L. The Laboratory Small Ruminant. Florida: CRC Press LLC, 1999. p.49-98.

BICUDO, S.D.; SOUSA, D.B.; TAKADA, L. Possibilidades e limitações da inseminação com sêmen ovino refrigerado e biotécnias associadas como estratégia de intensificação do manejo reprodutivo. Arc. Vet. Sci., v.5, p.35-39, 2000.

BRUN, M.V.; BARCELLOS, H.H.A.; OLIVEIRA, R.P. et al. Inseminação intra-uterina laparoscópica em ovinos utilizando agulha espinhal. In: CONGRESSO ESTADUAL DE MEDICINA VETERINÁRIA, 17., 2006, Gramado. Anais... Gramado, 2006. (Resumo).

HILL, J.R.; THOMPSON, J.A.; PERKINS, N.R. et al. Factors affecting pregnancy rates following laparoscopis insemination of 28,447 Merino ewes under comercial conditions: a survery. Theriogenology, v.49, p.697-709, 1998.

HONTOU, G.D. Inseminacíon artificial intrauterina mediante laparoscopia (IAI). In: DEL CAMPO, A.D. Manual práctico de reproducción e inseminacíon artificial en ovinos. Montevideo: Agropecuaria Hemisferio Sur, s/d. p.141-164, 2002.

LEMOS, S.L.S.; VINHA J. M.; SILVA, L.S. et al. Efeitos do pneumoperitônio com ar e $\mathrm{CO} 2$ na gasometria de suínos. Acta Cir. Bras., v.18, p.445-451, 2003.
MORGAN, G.E Jr. Anesthetic considerations. In: Minimally invasive surgery of the foregut. St Louis: Quality Medical Publishing, 1994. p.14-22.

OLIVEIRA, M.E.F. Técnicas de inseminação artificial em ovinos e caprinos. Acessado em 19 jun. 2009. Online. Disponível em: http://www.farmpoint.com.br/tecnicas-deinseminacao-artificial-em-ovinos-ecaprinos_noticia_52391_3_30_.aspx

ORTEGA, A.E; PETERS, J.H. Physiologic alterations of endosugery. In: Minimally invasive surgery of the foregut. St Louis: Quality Medical Publishing, 1994. p.23-37.

SALAMON, S.; MAXWELL, W.M.C. Frozen atorage of semen. II. Causes of low fertility after cervical insemination and methods of improvement. Anim. Reprod. Sci., v.38, p.1-36, 1995.

SILVA, L.C.; STOPIGLIA, A,J; FANTONI, D.T. Técnica de biópsia hepática em equino por laparoscopia. Cienc. Rural, v.32, p.459-465, 2001.

SILVA, A.E. Morfologia do orifício externo da cervix de ovelhas santa Inês. Disponível em: http://www.feinco.com.br/downloads_congresso/ 1268142743_262.pdf. Acessado em: 28 jun. 2009.

STELLFLUG, J.N.; WULSTER-RADCLIFFE M.C.; HENSLEY, E.L. et al. Oxytocin-induced cervical dilatation and cervical manipulation in sheep: effects on laparoscopic artificial insemination. J. Am. Sci., v.79, p.568-573, 2001.

VANNOZZI, I. Laparoscopic cryptorchidectomy in a cat- case report. J. Feline Med. and Surgery, v.4, p.201-203. 2002. 